\title{
Ludwig Schmugge
}

\author{
„Deus lo vult?“ \\ Zu den Wandlungen der Kreuzzugsidee im Mittelalter
}

\begin{abstract}
1. Einleitung
Friedensethik und Friedenstheologie der abendländischen Christenheit schließen es aus, so formulierte Klaus Schreiner, im Namen Gottes und im Interesse der christlichen Kirchen noch Kriege zu führen ${ }^{1}$. Dem war nicht immer so. Seit dem Ende des 11.Jahrhunderts wurde die Befreiung des Heiligen Grabes und der Stätten, „wo einst Jesus gewandelt ist“ (ubi steterunt pedes eius), seit 638 unter muslimischer Kontrolle, zu einer, wie Schreiner sagt, „Identitätsfrage der Christenheit“. Die Kreuzzüge, die als bewaffnete Pilgerfahrten begannen, erhielten bald durch die Päpste propagiert den Nimbus „Heiliger Kriege“. Kreuzzugsgelübde, Ablaß und Sündenvergebung bildeten die Vehikel, Rituale und Attraktionen dieses Phänomens „Kreuzzug“, für das es vor der Mitte des 13.Jahrhunderts nicht einmal ein lateinisches Wort gab. Doch der Kreuzzugsgedanke entfaltete seit dem Ersten Kreuzzug eine Eigendynamik, er wurde sich wandelnden historischen Situationen angepaßt, er war nie homogen und keinesfalls mit einer allseits akzeptierten Kriegsideologie identisch. Kritik und Widerspruch wurde bereits bei den Zeitgenossen laut. Gleichwohl fand das Wort „Kreuzzug“ als politischer Kampfbegriff universale Verwendung, bis in unsere Tage. Im 19. Jahrhundert, dem Zeitalter imperialer französischer Orientpolitik, nationalstaatlicher Stauferverklärung und preußisch-deutschen „Platz an der Sonne“Ehrgeizes führte der Herren eigener Geist nicht selten auch den Griffel der Kreuzzugshistoriker. Erst nach dem Zweiten Weltkrieg kam es allmählich zu einer Neubewertung.

Marcel Beck sah in den Kreuzzügen bereits lange vor israelischen Historikern wie Joshua Prawer $(+1990)$ den Versuch, ein koloniales Imperium zu errichten. Die negativen Folgen seien beträchtlich gewesen und hätten vor allem darin bestanden, daß „Europa wohl früher seine überragende Bedeutung erlangt hätte, wenn der Versuch ausgeblieben wäre, das östliche Mittelmeer zurückzugewinnen“2. Prawer kam indes später zu der Einsicht, der Erste Kreuzzug und

1 Siehe oben Einleitung S. VII.

2 Beck, Die geschichtliche Bedeutung 119.
\end{abstract}


die Eroberung Jerusalems seien keine kolonialistische Okkupation, sondern vielmehr eine Art „Dekolonisation“, eine legitime Wiederinbesitznahme heiliger Stätten der Christenheit. Anders das Urteil des Linkskatholiken und Amateur-Historikers Friedrich Heer: „Die Kreuzzüge, mit denen das christliche Abendland zwischen 1096 und 1270 das ,Heilige Land' zu erobern und den Vorderen Orient zu missionieren suchte, rechtfertigten sich religiös mit dem Hinweis auf die allein seligmachende Wahrheit des Christentums. ... Diese von den Päpsten propagierten und protegierten Kreuzzüge haben unendliches Unheil gestiftet."

Als Belege für den noch heute geläufigen weltanschaulichen, politischen bzw. sozialen Kampfbegriff „Kreuzzug“ seien genannt: „Kreuzzug gegen Hunger und Armut“, „Crusade for democracy“ (Woodrow Wilson), „Kreuzzug gegen den Analphabetismus“, „Kreuzzug gegen den Bolschewismus“ (Franco-Spanien) und schließlich die von Bush senior und junior propagierten „Kreuzzüge“ gegen den Irak Sadam Husseins.

Im folgenden sollen die Kreuzzüge nicht in die Typologie des „Heiligen Krieges" eingeordnet werden, wie das jüngst Egon Flaig getan hat, sondern zuerst soll der für Nicht-Mediävisten etwas rätselhafte Titel des Referats erklärt werden. Nach den „Gesta Francorum“, einer um 1100 aufgeschrieben Chronik des Ersten Kreuzzuges, soll „Gott will es“ (Deus le volt) der Schlachtruf der französischen Ritter gewesen sein, als sie auf dem Konzil von Clermont das Kreuz nahmen. Der Ruf wurde von der späteren französischen Historiographie dankbar aufgenommen: Kreuzfahrer waren (nach Lacroix) Ausführende einer gottgewollten Heilsgeschichte, Deus lo vult wurde so zu einem vorgeblichen Ausdruck der „theologie populaire“ jener Zeit.

\section{Die Fakten der Eroberung Jerusalems}

Im Vorderen Orient herrscht seit der Mitte des 11.Jahrhunderts so etwas wie ein geopolitisches Machtvakuum zum Nachteil des byzantinischen Reiches. Armenier, Petschenegen und andere Turkvölker rennen gegen die kleinasiatischen byzantinischen Territorien an, gefährden aber zugleich auch die Macht des Kalifen. Nach der Schlacht bei Manzikert 1071 kommt es zu einer Abwertung des Besant, des „Dollars des frühen Mittelalters“ (Roberto Sabbatino Lopez). Die seit dem frühen Mittelalter nie gänzlich abgerissenen christlichen Pilgerfahrten nach Jerusalem wurden mehr und mehr behindert, Wallfahrer schikaniert. 1065 gelangt eine Gruppe von mehreren hundert deutschen Pilgern nur nach heftigen Scharmützeln zum Heiligen Grab. Unter den vier Reichsbischöfen, die lieber im Sattel unterwegs waren als auf der Kanzel zu stehen, zeichnet sich der als blonder Hühne beschriebene Bamberger Oberhirte Gunther als besonders schlagkräftig aus. Als „Vorkreuzzug“ darf dieses Unternehmen aber nicht angesehen werden, die Bischöfe hatten vom deutschen König Heinrich IV. die Erlaubnis zu dieser Pilgerfahrt erbeten, nicht jedoch vom 
Papst $^{3}$. Als die Seldschucken 1078 Jerusalem in ihre Hand bekamen und 1084 auch das byzantinische Antiochia eroberten, wurden die Pilgerfahrten nach Jerusalem immer stärker behindert. Graf Robert von Flandern mußte sich 1089 seinen Weg zum Heiligen Grab durch Anatolien nach Jerusalem bereits freikämpfen.

Auf der von islamischen Machthabern beherrschten iberischen Halbinsel dagegen schreitet die Expansion christlicher Herrschaft, die Reconquista, erfolgreich voran. Eine wichtige Etappe stellte 1063 die Eroberung von Barbastro dar. Auch die italienischen Handelsmächte nutzen die Gelegenheit: Pisaner und Genueser Schiffe unter Beteiligung Amalfis greifen 1087 ins westliche Mittelmeer aus und überfallen die Stadt Mahdia im heutigen Tunesien. Ein Pisaner Kleriker berichtete darüber unmittelbar nach Ende der Aktion in einem "Gedicht über den Sieg der Pisaner“. Die Markgräfin Mathilde von Tuszien, enge Verbündete Papst Gregors VII., unterstützt diese Kampagne ebenfalls, der Bischof von Modena begleitet den Zug als päpstlicher Legat. Die Aktion gegen Mahdia kommt einem Kreuzzug bereits sehr nahe, zumal den Teilnehmern offenbar auch ein Ablaß in Aussicht gestellt worden war ${ }^{4}$.

Hilfe für den byzantinischen Basileus und Sicherung der Pilgerwege nach Jerusalem waren am Ende des 11. Jahrhunderts also das Gebot der Stunde auch aus Sicht der Päpste. Im Register Gregors VII. liest man zum Jahre 1074: „Alle sollen wissen, daß wir uns mit allen Mitteln bemühen werden, mit Gottes Hilfe dem christlichen Kaiserreich [Byzanz] zu Hilfe zu eilen. "5 Wenig später machte Papst Urban II. dem byzantinischen Kaiser Alexios (1081-1118) eine Zusage für militärische Unterstützung. 1095 rief derselbe Papst „zur Befreiung der Kirche im Osten auf“ (ad liberationem orientalium ecclesiarum) und das Konzil von Clermont sicherte den Teilnehmern einer solchen militärischen Aktion im Kanon 2 den Erlaß der Sünden zu: „Die Teilnahme am Marsch zur Befreiung der Kirche des Herrn in Jerusalem wird als vollständige Bußleistung angerechnet" (ad liberandam ecclesiam dei Ierusalem ... iter... pro omni poenitentia reputetur). 1099 wird Jerusalem erobert, aber nicht Byzanz profitiert vom Ersten Kreuzzug und erhält wie versprochen seine Territorien zurück, sondern westliche Fürsten errichten christliche Herrschaften in Antiochia, Edessa und Tripolis und trotz anfänglicher theologischer Bedenken ein Königreich Jerusalem. Die italienischen Handelstädte gewinnen im Heiligen Land Stützpunkte für ihre kommerziellen Aktivitäten. Eine dünne Oberschicht von Christen beherrschte seither die einheimische Bevölkerung von den Burgen und Städten aus.

3 Der Bericht über diese Pilgerfahrt bei dem Chronisten Lampert von Hersfeld und dem Annalisten aus dem Kloster Altaich, der Brief an Heinrich IV. MGH, Briefe der deutschen Kaiserzeit, Bd. 5 (Hannover 1950) Brief 23, 217-220.

4 Die Edition bei H.E.J. Cowdrey, The Mahdia campaign, in: English Historical Review 92 (1977) $1-29$.

5 Scitote nos ... omnibus modis id agere ..., ut adiutorium christiano imperio ... Deo iuvante faciamus, Register Gregors VII. I, 49. 


\section{Die Fakten des Zweiten und Dritten Kreuzzuges}

Keine 50 Jahre später kam mit der Eroberung Edessas durch den türkischen Heerführer Imad-ad-Din Zengi von Mossul am 24. November 1144 bereits das Ende vom Traum eines christlichen Morgenlandes. Die Zisterzienser Eugen III. und Bernhard von Clairvaux, die den unüberlegt unternommenen Zweiten „Kreuzzug“ $\mathrm{zu}$ verantworten hatten, führten sein Scheitern nicht auf die Übermacht Nur-ad-Dins vor Damaskus, sondern auf die Sünden des Abendlandes zurück: peccatis exigentibus lautete die fortan für alle Niederlagen der Christen schnell vorgebrachte Entschuldigung. Bei weitem nicht alle Zeitgenossen in Europa teilten diese Ansicht: Ein Würzburger Annalist schmähte Bernhard, „die Chimäre des Jahrhunderts", als Pseudopropheten ${ }^{6}$, und Gerhoh, Propst von Reichersberg, führte den Zweiten Kreuzzug, die "beklagenswerte Expedition“ (calamitosa expeditio) gegen Damaskus, auf die Habgier der Kreuzfahrer zurück ${ }^{7}$. Die schmale Oberschicht von maximal 150000 Christen, 120000 davon in den Städten lebend, konnte die Kreuzfahrerstaaten mit ihren ungeschützten Grenzen im Osten auf die Dauer auch mit Hilfe der neu gegründeten Ritterorden nicht halten.

Der „Heilige Krieg“ machte indes im 12.Jahrhundert auch in Europa Schule, ließ sich doch der Kreuzzugsgedanke in Zukunft für ganz unterschiedliche Ziele und Zwecke nutzbar machen. Nicht nur die Eroberung des Heiligen Landes, sondern jeder Krieg gegen „Heiden“ und später vor allem die Bekämpfung von Ketzern wurde durch Ablässe und Heilszusagen attraktiv gemacht. Der Polenherzog Boleslaw III. (1085-1138) ließ für die von ihm unternommene Unterwerfung der slawischen Stämme zwischen Elbe und Oder durch den Erzbischof von Magdeburg nach dem Vorbild des Ersten Kreuzzuges einen „Heiligen Krieg“ ausrufen. Die norddeutschen Fürsten Heinrich der Löwe (1130-1195), Albrecht der Bär (1100-1170) und einige Bischöfe des Reiches ließen ihre Eroberungspläne vom Papst als Kreuzzugsersatz absegnen. Statt mit in den Orient zu ziehen, eroberten sie mit päpstlichem Segen als „Kreuzfahrer“ lieber christliche Siedlungen vor ihrer „Haustür“, im Wendenland und in Pommern. Im Westen des Mittelmeeres führte Genua parallel zum Zweiten Kreuzzug 1146-48 seinen „Kreuzzug“ gegen Minosca und Almerìa auf der iberischen Halbinsel, der in erster Linie von Handelsinteressen bestimmt war.

Der Normannenkönig Roger II. von Sizilien wollte von Eugen III. sogar die Zustimmung für einen Kreuzzug gegen Byzanz erhalten. Zur Eroberung von Konstantinopel kam es allerdings erst zwei Generationen später. Die perverse Idee von „Kreuzzügen“ gegen Christen, die seit dem 13.Jahrhundert an der Tagesordnung waren, kündigt sich hier bereits unheilvoll an. Der einzige dauernde Erfolg wurde 1147 von kölnischen, flämischen und englischen Kreuzfahrern erzielt, die Lissabon eroberten, was ihnen mit den Worten des Chronisten Osbern

${ }^{6}$ MGH SS 16 S. 3.

7 De investigatione antichristi, MGH Libelli de lite III S. 374. 
unter Hinweis auf einschlägige Stellen aus Yvo von Chartres „Panormia“, einer Rechtssammlung, als ein „gerechter Krieg“ (bellum iustum) dargestellt wurde.

In diesem Kontext gilt es, kurz bei der rechtlichen Begründung der ersten Kreuzzüge zu verweilen. Es ist bemerkenswert, daß Gratian, der Schöpfer des systematischen Kirchenrechts, an keiner Stelle seines um 1140 abgeschlossenen und in zwei Fassungen überlieferten Dekrets den Kreuzzug diskutiert ${ }^{8}$. In Causa 23 behandelt er allerdings, auf Augustinus basierend, die Frage, was die Kennzeichen eines gerechten Krieges seien ${ }^{9}$. Die Voraussetzungen für ein bellum iustum bestanden nach Gratian neben der gerechten Sache in der notwendigen „Autorität“ (auctoritas), die einen Krieg anordnen konnte. Unstrittig waren für den Rechtsgelehrten die vom Herrn befohlenen Kriege des Alten Testaments heilig. Doch dem Phänomen Kreuzzüge steht nicht nur Gratian, sondern auch die Dekretistik fremd gegenüber. Es ist ein Faktum, daß keine der päpstlichen Kreuzzugsbullen des 12. Jahrhunderts in den „Liber extra“, die epochemachende Sammlung der päpstlichen Dekretalen, von Gregor IX. 1234 promulgiert, aufgenommen worden ist ${ }^{10}$. Die Dekretisten waren wie Gratian der Ansicht, daß - abgesehen von der legitimen Selbstverteidigung gemäß der römisch-rechtlichen Maxime: „Es ist Rechtens Gewalt mit Gewalt zu vergelten“ (vim vi repellere fas est) - jeder kriegerische Akt autoritärer Erlaubnis bedürfe. Aber wer war außer dem Herrgott sonst noch berechtigt, einen Krieg für gerecht zu erklären? Der Kardinal Hostiensis und Papst Innozenz IV. schrieben auf der Basis der hierokratischen Theorie allein dem Papst das Recht zu, Kreuzzüge auszurufen und zum bellum iustum zu erklären ${ }^{11}$. Eine Mehrheit der 1200 Teilnehmer des IV. Laterankonzils 1215 scheint der Theorie vom „Heiligen Krieg“ dagegen eher skeptisch gegenübergestanden zu haben. Im entsprechenden Kanon mit dem Aufruf zur Befreiung des Heiligen Landes, den die Konzilsväter erlassen haben, ist von einem bellum iustum keine Rede ${ }^{12}$.

\section{Kreuzzugskritik}

Mit dem Scheitern des von Bernhard von Clairvaux propagierten Zweiten Kreuzzuges verschafft sich in der Christenheit eine nie mehr verstummende Kritik an den Kreuzzügen Gehör. Wie der bereits zitierte Würzburger Annalist bemerkte, hätten „Pseudopropheten, Söhne Belials, Zeugen des Antichrists“ durch „haltlose Versprechungen“ (vana predicatione) die Christen gegen die Sarazenen zu ziehen

8 Dazu Russell, The Just War 83; Hehl, Kirche und Krieg 57-108.

9 Decretum Gratiani C. 23 q. 2 c. 1: Quid sit iustum bellum; C. 23 q. 2 c. 2: iusta bella solent diffiniri, que ulsciscuntur iniurias ... vel reddere quod per iniurias ablatum est. Ed. Friedberg, Sp. 894.

10 Es handelt sich um Eis qui Hierosolymam Papst Calixts II., 1123, Lateran I c. 10; Quantum predecessores Eugens III. bis hin zu Ad liberandam Innozenz III.

11 Russell nennt diese Haltung „theocratic megalomania“, 297; vgl. dazu auch Hehl, Kirche und Krieg $251 \mathrm{ff}$.

12 IV. Laterankonzil c. 71: Ad liberandam terram sanctam. 
bewogen. Neugier und Gewinnsucht und nicht der Eifer für den Herrn hätten die Menschen angetrieben ${ }^{13}$. Jede theologische Berechtigung des Kreuzzugsablasses wird hier schlichtweg bestritten. Die umfassendste Kreuzzugskritik wurde von dem englischen Gelehrten Radulfus Niger, einem Juristen, Theologen und Historiker, der an der Universität Paris gelehrt hatte, kurz nach dem Fall Jerusalems 1187 vorgebracht. Sein Traktat „Über das Kriegswesen und den dreifachen Pilgerweg nach Jerusalem" (De re militari et triplici via peregrinationis hierosolymitana) ist dem französischen König Philipp Augustus gewidmet, noch bevor der zum Dritten Kreuzzug aufbrach ${ }^{14}$. Sie kommt sozusagen „von rechts“, von einem überzeugten Hierokraten, und basiert auf einer umfassenden Gesellschaftskritik. Welches sind die Kernpunkte der Argumentation Nigers?

1. Das Heilige Land (ausgenommen die Ritterorden) sei ein Schmelztiegel abendländischer Kriminalität, luxuria herrsche hier vor.

2. In der europäischen Christenheit grassierten zahlreiche Häresien. Bei der Vorbereitung des III. Laterankonzils 1179 seien in der Lombardei 19 und in Frankreich 50 verschiedene Sekten ausgemacht worden, noch mehr davon in England. Die Katharer, Waldenser sowie die Bogumilen könnten hier gemeint sein.

3. Der Klerus sei verrottet, von Ehrgeiz, Habsucht und Nepotismus geleitet, der Konkubinat unter den Geistlichen weit verbreitet. Die Mönche werden von der Schelte Nigers nicht ausgenommen. Simonie sei überall in der Kirche an der Tagesordnung.

4. Die Laien, insbesondere die Fürsten, seien um keinen Deut besser als die Geistlichen. Auch sie betrieben Simonie und Nepotismus und setzten das weltliche Schwert durch ungerechte Steuern und Münzverrufung schamlos zu ihrer Bereicherung ein. Auch den seit Papst Innozenz III. üblichen Kreuzzugszehnten zählt Niger zu diesen illegalen Maßnahmen. Besonders gegen die unter den Rittern so beliebten (aber von der Kirche verbotenen) Turniere wettert er. Mit luxuria und offenem Ehebruch brüsteten sich die Adligen. Auch Kaufleute schreckten vor Betrug nicht zurück, Richter und Advokaten seien bestechlich.

Wie schon der Würzburger Annalist lehnt Niger den Kreuzzugsablaß als bußtheologisch unhaltbar ab. Solange ein Kreuzfahrer seine Sünden nicht bereue, dafür gebüßt und angerichteten Schaden wiedergutgemacht habe, sei alle Hoffnung auf Sündenvergebung durch einen Ablaß vergebens. Statt einer realen Kreuzfahrt ins Heilige Land empfiehlt er die „mystische Pilgerfahrt“ (mystica peregrinatio) in das spirituelle Jerusalem. Der Papst, so behauptet Niger, „rate zur Kreuzfahrt und verspreche den Kreuzfahrern den Nachlaß aller Sünden“ (remissionem omnium suorum peccatorum). Das stand zwar so verkürzt nicht in der Kreuzzugsbulle „Audita tremendi“ Papst Gregors VIII. von 1187, aber es war sicher die populäre Formel, mit welcher die Prediger den potentiellen Kreuzfahrern, die ja fast alle Laien waren, die Teilnahme schmackhaft gemacht haben dürften. Für Niger sind

13 MGH SS 16 S. 3.

14 Radulphus Niger, De re militari, ed. Schmugge (Beiträge zur Geschichte und Quellenkunde des Mittelalters 6, Berlin 1977). 
solche Kreuzzugsprediger (und damit auch der Papst) „falsche Propheten“. Nicht nur die Sündenstrafen, sondern schlichtweg alle Sünden sollten vergeben sein. Die Bischöfe von Paris, Reims und Sens, denen er seine Schrift zur Zensur vorgelegt hatte, haben an Nigers Ausführungen offenbar keinen Anstoß genommen.

Ein Kreuzzug verstößt nach Nigers Meinung nicht nur gegen die Grundsätze der Bußtheologie, sondern auch gegen das evangelische Missionsgebot. Die Kirche dürfe auf keinen Fall zum Blutvergießen beitragen. Eine Rechtfertigung des Kreuzzuges als bellum iustum kommt für ihn nicht in Frage, denn der Glaube dürfe nicht mit dem Schwert verkündet, das Christentum nur aus freien Stücken angenommen werden. Hier zeigt sich bereits der noch zu behandelnde franziskanische Missionsgedanke. Daß Niger indes kein Pazifist war, geht schon daraus hervor, daß er das „weltliche Schwert“ (gladius materialis) ohne Zögern gegen Ketzer einsetzen will. Von den Ketzern nämlich drohe seiner Ansicht nach den Christen eine reale Gefahr, nicht von den Sarazenen. Während der Abwesenheit zahlreicher Fürsten und Ritter auf einem Kreuzzug könne es gar zu einem bewaffneten Aufstand der Ketzer gegen die Kirche kommen, so befürchtet Radulfus Niger, zu einer Revolution der Häresie.

Auch die vox populi schnödet im Laufe des 13. Jahrhunderts gegen die Kreuzzüge: „Nicht [allein] in Jerusalem gewesen zu sein, sondern in Jerusalem ein gutes Leben geführt zu haben, ist verdienstvoll“ (Non Ierosolimis fuisse, sed Ierosolimis bene vixisse laudandum est), hatte bereits Hieronymus gegen die zu seiner Zeit, im 5. Jahrhundert, beliebten Jerusalempilgerfahrten eingewandt. Der Vers wird im Mittelalter häufig zitiert, auch in Gratians Dekret kommt er vor ${ }^{15}$. Ferner dichtet der Volksmund: „Ein Kreuz auf dem Mantel macht noch keinen guten Menschen“ (Crux in mantellis non est purgatio pellis) oder „Das Kreuz auf dem Mantel bringt noch keine Verdienste“ (Crux vestimenti dat premia nulla ferenti). Selbst der Vers aus den Episteln des Horaz, Caelum non animum mutant, qui trans mare currunt (frei übersetzt: „Wer übers Meer fährt, wechselt das Klima, nicht seinen Geist“), wird jetzt auf den Kreuzzug bezogen ${ }^{16}$. Ein Annalist aus Stade, der den HorazVers zitiert, bemerkt erklärend, der Satz treffe zu, denn er habe noch keinen Kreuzfahrer getroffen, der aus dem Heiligen Land geläutert zurückgekehrt sei ${ }^{17}$.

Derartige kreuzzugskritische Stimmen haben bereits im 12.Jahrhundert bei Klerikern wie Laien ein positives Echo gefunden. Keineswegs alle Zeitgenossen waren davon überzeugt, daß die Kreuzzüge eine primäre Aufgabe der Kirche seien. Bereits der große Kirchenrechtler Bischof Yvo von Chartres (+1117) hatte den Grafen Hugo von Trois von der Teilnahme abgehalten. Ebenso war der englische König Heinrich II. dem Rat seiner Bischöfe gefolgt und hatte aus Gründen der Staatsräson nicht selbst das Kreuz genommen. Als Kreuzfahrer würde er, argumentierten seine Berater, seine erste Pflicht, Frieden und Recht in seinem Reich zu garantieren, nicht erfüllen können. Erzbischof Wilhelm von Reims, Statthalter

${ }^{15}$ Decretum Gratiani C. 12 q. 2 c. 71 , Friedberg, Sp. 711.

16 Horaz, Ep. I, 11,27.

17 Annales Stadenses MGH SS XVI S. 344. 
Philipps II. Augustus während dessen Kreuzzug, zog es vor, nach der Rückkehr des Königs nach Santiago zu pilgern und nicht nach Jerusalem. Der Pariser Theologe Alanus ab Insulis (+1203) verkündete in einer Predigt, die Kreuznahme sei frivol und nutzlos für den Menschen, wenn ihr nicht eine innere Umkehr vorausgehe. Ähnliche Gedanken sind zur gleichen Zeit auch bei dem Zisterzienser Joachim von Fiore $(+1202)$ zu finden, der sich äußerst kritisch gegen die diversen päpstlichen Kreuzzugspläne des 13.Jahrhunderts geäußert hat.

\section{Kreuzzüge im 13. und 14. Jahrhundert}

Doch zurück zur faktischen Kreuzzugsgeschichte. Sultan Saladin hatte nach der Schlacht bei Hattin am 2. Oktober 1187 Jerusalem nach 88 Jahren den Christen wieder entrissen. Bis auf die Küstenstädte Antiochia, Tripolis und Tyrus verloren die Franken alle ihre Siedlungen. Die Bulle mit den Anfangsworten „Audita tremendi“ vom Ende des Jahres 1187 führte die Niederlage erneut auf die Sünden der gesamten Christenheit zurück, nach Riley-Smith ein Zeichen der „low moral“ des Westens ${ }^{18}$. Daß christliche Ritter wie Rainald von Châtillon hemmungslos Friedensverträge mit den Sarazenen brachen und deren Karawanen, die auf der Handelsstraße von Damaskus nach Kairo zogen, überfielen und ausraubten, war den westlichen Historikern keine Notiz wert. Der 1189 begonnene, von großen Hoffnungen begleitete Dritte Kreuzzug scheiterte unter anderem auch an der verfehlten logistischen Entscheidung, das Heer Friedrich Barbarossas über Land gegen die Muslime zu führen, sowie am Mißtrauen zwischen Richard Löwenherz und Philipp II. Augustus von Frankreich. 1204 schließlich desavouierte in erster Linie der venezianische Handelsegoismus den religiös motivierten Kreuzzugsgedanken mit der Eroberung der ungarischen Stadt Zara und schließlich der byzantinischen Hauptstadt Konstantinopel. Dorthin war das Kreuzfahrerheer auf den venezianischen Schiffen „umgeleitet" worden. Zwar exkommunizierte Innozenz III. die Kreuzfahrer, aber die Plünderung Konstantinopels konnte er nicht verhindern. Deshalb versuchte der Papst ein neues Kreuzzugsunternehmen in Gang zu bringen, indem er durch Kreuzzugssteuern die gesamte Kirche einbezog.

Doch der von ihm minutiös vorbereitete Kreuzzug war mit dem Tode des Heiligen Vaters am 16.Juli 1216, der sich selbst an die Spitze hatte stellen wollen, praktisch gescheitert. König Andreas von Ungarn und Herzog Leopold von Österreich, die durch einen Angriff auf Damiette im Nildelta die Macht des Kairoer Ayubiten-Sultans zu brechen suchten, konnten sich gegen den Starrsinn des päpstlichen Legaten Pelagius, der das Angebot einer Rückgabe Jerusalems durch Sultan al-Kamil zurückgewiesen hatte, nicht durchsetzen. Auch Innozenz III. hatte bereits versucht, den Sultan durch Verhandlungen dazu zu bewegen, den Christen das Heilige Land und Jerusalem zu überlassen. Der Kreuzzug gegen Damiette stellte gleichwohl eine Wende dar. Franz von Assisi hatte nämlich zeitweilig an

18 Riley-Smith, The Crusades 104. 
diesem Unternehmen teilgenommen. In klarer Ablehnung aller Politik einer Zwangsbekehrung und der Mission mit dem Schwert vertraute der Gründer des Minoritenordens auf das Wort des Evangeliums. Bereits 1211 und 1213/14 hatte er den Versuch unternommen, den Muslimen in Syrien und Marokko den Glauben $\mathrm{zu}$ predigen. So wanderte er von Damiette aus mit einem Begleiter und einem Dolmetscher nach Kairo, trat vor Sultan al-Kamil und gab sich alle Mühe, ihn zur Annahme des christlichen Glaubens zu bewegen. Zwar hatten seine Worte keinen Erfolg, aber das Prinzip der friedlichen Mission war geboren.

Derweilen schien der Antichrist, wie Radulfus Niger befürchtet hatte, auch im Innern der Christenheit auf dem Vormarsch. Die sich ausbreitenden Häresien (Waldenser, Katharer, Bogumilen) waren durch die vom III. Laterankonzil initiierten Predigt- und Missionskampagnen nicht zu bannen. Kaiser und Papst hatten daher zu härteren Mitteln gegriffen, und gemeinsam hatten sie in einer Bulle („Ad abolendam“) bereits 1184 den Einsatz auch des weltlichen Arms gegen die Ketzer gefordert. Der Albigenserkrieg (1208-1229) war die traurige Konsequenz, aber auch die logische Weiterentwicklung des Kreuzzugsgedankens. Es ist bezeichnend, daß der schärfste Kritiker des Kreuzzuges gegen Konstantinopel, Simon von Montfort, keine Bedenken hatte, den Ketzerkreuzzug in Südfrankreich anzuführen. Noch Innozenz III. hatte den Legaten Peter von Castelnau beauftragt, gegen die katharischen Gemeinden im Gebiet von Toulouse zu predigen, und den Grafen Raimund exkommuniziert. Als Peter 1208 ermordet wurde, rief der Papst einen Kreuzzug aus, den ersten Kreuzzug in einem christlichen Land, und übertrug dem Grafen Simon von Montfort den Oberbefehl. Die zahlreich ins Land strömenden Ritter richteten 1209 in Béziers und später in Albi ein Blutbad an und verschonten weder Katholiken noch Katharer, denn sie waren aufgefordert, „alle zu töten, denn der Herr werde die Seinen erkennen“.

Nicht nur dem Grafen Raimund von Toulouse, auch dem deutschen Kaiser Friedrich II., der bereits 1215 persönlich das Kreuz genommen hatte, wurde der Kreuzzug zum Verhängnis. Nachdem er das Unternehmen immer wieder hinausgeschoben hatte und deshalb schließlich von Papst Gregor IX. gebannt worden war, trat Friedrich Ende Juli 1228 die Überfahrt nach Akkon an. Ein Jahr zuvor hatte ihm Sultan al-Kamil einen Vertrag über die Rückgabe Jerusalems angeboten. Ohne einen Schwertstreich erlangte der Kaiser, vorerst für zehn Jahre, die Herrschaft über die Heilige Stadt und krönte sich in der Grabeskirche vermutlich selbst zum König von Jerusalem. „Eine bonapartistische Selbstdarstellung und eine Zurückweisung aller kirchlicher Ansprüche über Jerusalem“ (so Kantorowicz)? Wohl eher nicht. Es überforderte allerdings die damaligen Vorstellungen von religiöser Toleranz, daß in dem Vertrag den Muslimen die religiöse Nutzung des Felsendomes zugestanden worden war. Dem Kaiser wurde folglich ein impium foedus mit den Sarazenen vorgeworfen, er wurde zum Verräter des Glaubens gestempelt.

Auf dem Konzil von Lyon 1245, als Jerusalem bereits wieder in den Händen der Ayubiten war, wurde der Bann über ihn erneuert und nie mehr aufgehoben. Die Päpste setzten die Waffe des Kreuzzugs nun gegen den Kaiser und das „Geschlecht 
der Kirchenverfolger“ (genus persecutorum), nämlich alle Staufer, ein. Sie wiederholten die Aufrufe zum Kreuzzug mit schöner Regelmäßigkeit, bis schließlich nach Tagliacozzo 1268 mit Konradin der letzte legitime Staufer beseitigt war. Auch nach der Sizilianischen Vesper am 30. März 1282 wurde in Frankreich ein Kreuzzug gegen Peter von Aragon gepredigt, der die Anjou von der Insel verjagt hatte. Diese von den Päpsten proklamierten „politischen Kreuzzüge“ konnten nicht ohne Folgen bleiben. In Italien entstand die „Halleluja-Friedensbewegung“, im Territorium des Reiches, so bemerkte der prostaufischer Haltung ganz unverdächtige Hostiensis in seiner vor 1253 abgeschlossenen „Summa aurea“ zum „Liber extra“, habe es massive Kritik an der päpstlichen Kreuzzugspolitik gegeben ${ }^{19}$. In der Tat findet, wie die von Hostiensis zitierten anonymen Deutschen richtig bemerkten, der Kreuzzug gegen „Schismatiker und Rebellen in der Kirche“ im kanonischen Recht keine Erwähnung.

Nicht nur die Deutschen wunderten sich. Als der päpstliche Legat Rostand ins Königreich England gesandt wurde und in London in den 1250er Jahren zum Kreuzzug gegen den Staufer Manfred aufrief, so berichtet der Chronist Mathew Paris, hätten die Gläubigen (mit echt englischem schwarzen Humor) sich erstaunt gezeigt, daß ihnen genau so viel für das Blut von Christen versprochen werde wie für das der Ungläubigen (nämlich der Nachlaß der Sünden), und sie hätten den Legaten und seine Prediger ausgelacht ${ }^{20}$. Nach dem Fall von Akkon 1291, der letzten christlichen Bastion im Heiligen Land, blieben die immer aufs neue geschmiedeten Pläne zur Rückeroberung des Heiligen Landes nur Makulatur. Allein auf der iberischen Halbinsel kam die Reconquista voran, doch weitgehend ohne militante Kreuzzugsideologie. Raimundus Lullus (1232-1316) hat nach seinen Erfahrungen als königlicher Seneschall auf dem kurz zuvor zurückeroberten Mallorca ebenfalls auf die Karte der friedlichen Mission gesetzt und sein Leben lang versucht, Muslime und Juden predigend zu bekehren. 1295 überreichte er dem Papst ein Memorandum, wie man dem Islam missionarisch begegnen könnte. Zehn Jahre später publizierte Lull einen „Liber de fine“, in welchem er die Idee einer Reconquista mit Hilfe des franziskanischen Missionsgedankens erneut ins Spiel brachte. Durch gut ausgebildete, arabisch sprechende Priester sollte Nordafrika bekehrt werden und so die christliche Herrschaft Schritt für Schritt wieder bis ins Heilige Land ausgedehnt werden. Lull wurde in Tunis auf einer Missionsreise gesteinigt und starb an den Folgen.

Das Konzil von Vienne 1311 regte in ähnlicher Absicht an, in Rom, Paris und Toledo Lehrstühle für orientalische Sprachen zu errichten, um Missionare gründlich auf ihre Tätigkeit vorzubereiten. Und während König Peter I. von Zypern aus in der Mitte des 14.Jahrhunderts militärische Aktionen gegen Südanatolien und Alexandria unternahm, kamen seit den 1330er Jahren die Pilgerfahrten nach

\footnotetext{
19 Summa Aurea III, 34, de voto $\$ 19$, Ausgabe Lyon 1537, S. 178va: in Alemannia vidi aliquos super hoc dubitare, asserentes quod contra christianos non videbatur equum et honestum, quod crux assumeretur maximeque quod non invenitur in iure expressum.

20 Rerum Britannicarum Scriptores V, 521.
} 
Jerusalem wieder in Gang, betreut durch die Franziskaner, die bis heute in Jerusalem ein Kloster unterhalten. Zur gleichen Zeit begannen die osmanischen Eroberungen in Kleinasien und auf dem Balkan, das Byzantinische Reich endgültig zu vernichten. Als die Osmanen um 1400 die Donau erreichten, wurde die Bedrohung des christlichen Europas handgreiflich, nur kurzzeitig durch die Mongolen Timur Lenks (+1405) unterbrochen. König Sigismund hob als erster „den Türkenzug auf die europäische ... Ebene ${ }^{\text {" } 21}$, denn die Schlacht bei Nikopolis hatte 1396 die militärische Überlegenheit der Türken nur zu deutlich gemacht. Man lese in seinem „reisbuch“ nach, wie der bayerische Knappe Johann Schiltberger diese dramatischen Jahre an allen Fronten durchlebte: Bei Nikopolis geriet der Kreuzfahrer in osmanische Gefangenschaft, 1402 bei Ankara als türkischer Söldner von Truppen Timur Lenks aufgegriffen, diente er bis 1426 verschiedenen Prinzen der Goldenen Horde in Asien, floh 1426 nach Konstantinopel und kam ein Jahr später nach 30 Jahren Abenteuer wieder in seine bayerische Heimat zurück ${ }^{22}$.

Schauplatz von Kreuzzügen war für die nächsten drei Jahrhunderte nicht der Vordere Orient, sondern Europa. Kreuzzugsaufrufe wurden seit Bonifaz IX. (1389-1404) wieder zur päpstlichen Routine. Als in der Schlacht von Varna (1444) das christliche Heer den Truppen Sultan Murads II. unterlag, zogen es Serbien und Venedig vor, einen Separatfrieden mit den Osmanen zu schließen, die jetzt den Balkan südlich der Donau kontrollierten. Sonderfrieden mit den Türken waren an der Tagesordnung, das Ende des Ostkaiserreichs war nur noch eine Frage der Zeit. Konstantinopel fiel bekanntlich 1453, während zeitgleich im Westen Kunst und Technik revolutioniert wurden: Dufay komponierte die vierstimmige Motette „Lamentatio matris ecclesiae Constantinopolitanae“, Gutenberg druckte die Kreuzzugsbulle Nikolaus V. Die Mauern der Stadt am Bosporus waren von drei in Ungarn gegossenen Riesenkanonen sturmreif geschossen worden. In der Christenheit fürchtete man trotz des Friedens von Lodi 1454, Mechmed der Eroberer werde bald auch Rom einnehmen. So bemühte sich vor allem Papst Pius II. auf dem Fürstentag zu Mantua um eine gemeinsame Aktion der europäischen Fürsten, mit kargem Erfolg. Dennoch stellte er sich 1464 selbst an die Spitze eines geplanten Kreuzzuges. Er starb in Ancona am 14. August 1464, noch bevor die Schiffe überhaupt lossegeln konnten. Der Friede in Europa sowie die Hoffnung, Konstantinopel und Jerusalem in einer Aktion zurückgewinnen zu können, blieben eine Illusion, denn die Bedrohung durch Mechmed II. nahm weiter zu: 1480 erlebt Otranto den 11. September des 15.Jahrhunderts. Bei einem im ausdrücklichen Auftrag Mechmeds des Eroberers durchgeführten türkischen Terror-Überfall auf die apulische Stadt wurden fast alle Einwohner, denen es nicht gelang, ins Hinterland zu fliehen, umgebracht. Zweihundert Jahre später stand ein türkisches Heer vor Wien.

21 Mertens, Europäischer Friede 66.

22 Ausgaben und Lit. in: Repertorium Fontium X (Rom 2005) 308-309. 


\section{Humanisten, Kreuzzug und Heiliger Krieg}

Es verwundert nicht, daß in den Zeiten stets wachsender Bedrohung durch den Halbmond der Blick zurück in die Geschichte gerichtet wurde. Die Historiographie des 15. und 16.Jahrhunderts zeigt, daß angesichts der Türkengefahr gerade die Humanisten den Kreuzzügen als der (vermeintlich) heroischen Zeit christlichen Rittertums ihre besondere Aufmerksamkeit widmeten. Die studia bumanitatis umfaßten nicht nur die Texte der antiken Autoren, sondern auch die Historie, vornehmlich die antike und die zeitgenössische. Die Geschichte des Mittelalters wurde dabei eher von ihrer negativen Seite betrachtet, mit einer großen Ausnahme, der Kreuzzüge. Fast alle humanistischen Geschichtsschreiber erklärten, wie wir sehen werden, die christlichen Glaubenskriege zu einer heiligen Sache und zu einem Vorbild für ihre Gegenwart. Sie hielten die Kreuzzüge des 12. und 13.Jahrhunderts dem von den Türken bedrohten 15.Jahrhundert als Spiegel vor.

Die Initialzündung für dieses Interesse ist durch das Jahr 1453 gegeben, wie an der Florentiner Historiographie schön zu erkennen ist. Leonardo Bruni (+1444) und Poggio Bracciolini (+1459) übergehen in ihren Geschichten der Stadt Florenz die Kreuzzüge noch mit Stillschweigen. Beginnend mit den „Dekaden“ Biondo Flavios (+1463) feiern die humanistischen Historiker die Kreuzzüge als eine gewaltige, den Päpsten zu verdankende Leistung des hohen Mittelalters. Der päpstliche Sekretär Biondo betätigte sich auch als politischer Publizist, indem er König Alfons von Aragon 1453 und ein Jahr später den Dogen von Venedig, Francesco Foscari, zu einem Kreuzzug gegen Mechmed II. aufforderte ${ }^{23}$. Bei seiner Darstellung des Zeitalters der Kreuzzüge ging Biondo nicht besonders quellenkritisch vor, so folgte er stets einer Leitquelle. Beim Ersten Kreuzzug vertraute er sich, wie bereits Jakob Burckhardt nachgewiesen hat, einer zweitrangigen Chronik an, derjenigen von Robert dem Mönch, für die weiteren Abschnitte Wilhelm von Tyrus. Am Scheideweg, Geschichte als Kunst - als rhetorische Kunst - oder als Handwerk zu betreiben, entschied sich der Humanist zwar für das letztere, entwickelte dabei jedoch keineswegs die den Humanisten ansonsten zugeschriebene systematische Quellenkritik.

Biondos „Dekaden“ „befruchteten“ die humanistische Historiographie. Aenea Silvio Piccolomini verfaßte als Papst 1463 eine Kurzfassung der ersten beiden Dekaden und trug damit mehr zur Verbreitung der Kreuzzugsidee bei als durch seine Predigten und Kongresse. Der Mailänder Lodrisio Crivelli, ein Freund Aenea Silvios aus den Tagen des Basler Konzils, stellte seinem Aufruf zu einer militärischen Aktion gegen die Türken ebenfalls einen Abriß der Geschichte der Kreuzzüge voran. Bei allem Säbelgerassel ging die Idee Franz von Assisis doch nicht ganz verloren. Immerhin stellte sich Aenea Silvio als Pius II. wenigstens versuchsweise in dessen Tradition, wenn er Mechmed den Eroberer 1461 brieflich zu bekehren versuchte. Der Nachfolger Bracciolinis im Amt des Florentiner Kanzlers, Benedetto

${ }^{23}$ Vgl. zu diesem Abschnitt Schmugge, Die Kreuzzüge 9ff. 
Accolti, widmete seinen Kreuzzugsaufruf, dem ebenfalls die Geschichte der christlichen Heldentaten im Heiligen Land verbunden war, seinem Herrn Piero de Medici. Doch auch Accolti faßte dabei nur das Werk des Wilhelm von Tyrus kurz zusammen. Als der päpstliche Bibliothekar Platina im Auftrag Sixtus IV. für das Heilige Jahr 1475 die Papstviten zusammenstellte, nutzte er für die KreuzzugsPäpste Urban II., Paschalis II., Eugen III. und den Dritten Kreuzzug einzig die „Dekaden“ des Biondo Flavio.

Gedruckte und geschriebene Geschichten des Heiligen Landes fanden wegen ihrer Aktualität reißenden Absatz, allein etwa 350 spätmittelalterliche Pilgerberichte von Jerusalemreisen liegen vor, teilweise auch als Inkunabeldrucke. Gutenbergs Erfindung der beweglichen Lettern nutzten die Drucker auch dazu, die Primärquellen der Kreuzzüge zu verbreiten. Die bereits genannte Geschichte des Ersten Kreuzzuges aus der Feder Roberts des Mönches liegt in mindestens 94 Handschriften vor. Antonin von Florenz (+1459) bezog in seiner Weltchronik die Informationen über die Kreuzzüge von Wilhelm von Tyrus, während der österreichische Geschichtsschreiber Thomas Ebendorfer in seinem Werk „Über die beiden Kreuzfahrten der christlichen Fürsten“ (De duobus passagïs christianorum principum, 1454-1456) seine Kenntnisse aus der Lektüre Roberts des Mönches bezogen hatte. Wir können also festhalten, daß in Italien in der Mitte des 15. Jahrhunderts durch die Türkenbedrohung und den Fall von Konstantinopel ein brennendes Interesse an der Geschichte der Kreuzzüge des späten 11. und 12. Jahrhunderts geweckt worden ist, das in zahlreichen Werken humanistischer Historiker vielfältigen Niederschlag fand.

Auch die berühmte Disputation Bartolomeo Platinas, des ersten Bibliothekars der Biblioteca Vaticana, in der Engelsburg mit Rodrigo Sánchez de Arévalo, dem päpstlichen Kastellan (1468), ist ein Beispiel dafür, daß Glaubenskriege aus der Diskussion über Krieg und Frieden bei den Humanisten nicht wegzudenken waren. Arévalo verteidigt die persönliche Teilnahme Pius II. am Kreuzzug, ja teilt dem Krieg eine „grundlegende heilsgeschichtliche Funktion“ zu. „Zur Verteidigung des christlichen Glaubens und zur Ausdehnung christlicher Herrschaft" sei der Krieg in jedem Fall gerechtfertigt ${ }^{24}$. Kardinal Bessarion, der die Folgen der Expansion des Halbmonds durch die Vertreibung aus Byzanz am eigenen Leibe erfahren hatte, schrieb 1470 nach der Eroberung von Negroponte seine berühmten Reden gegen die Türken und rief zur Verteidigung von Freiheit und Heimat auf ${ }^{25}$. Frieden unter den Christen sei die Voraussetzung für einen erfolgreichen Kreuzzug gegen die Türken. Mit dem Einmarsch Karls VIII. nach Italien 1494 wurde der Türkenzug allerdings zum Deckmantel für eine Hegemonialpolitik großen Stils in Europa.

Nördlich der Alpen wurde die Türkengefahr nicht weniger verspürt als am Mittelmeer, standen doch 1529 die Türken sogar vor Wien. Die Geschichtsschreiber der Zeit um 1500 (Sebastian Brandt, Ulrich von Hutten, Hartmann Schedel,

${ }^{24}$ Benzinger, Zur Theorie von Krieg und Frieden 141-149 und 158f.

${ }^{25}$ Dazu zuletzt Benzinger, Zur Theorie von Krieg und Frieden $185 \mathrm{f}$ mit der älteren Lit. 
Jacob Wimpfeling, Nauclerus und Cuspinian), insbesondere dann die Anhänger der Reformation, setzten mit ihrer fundamentalen Kirchen- und Papstkritik in der Geschichtsschreibung neue Akzente. Einen entscheidenden Aspekt protestantischer Geschichtsschreibung im Hinblick auf die Beurteilung der Kreuzzüge hat erstmals Ulrich von Hutten zur Sprache gebracht. In seinem Mahnbrief an die deutschen Fürsten, endlich einen Krieg gegen die Türken zu unternehmen (Exhortatio ad principes Germanos, ut bellum Turcis inferant), wirft er den Päpsten vor, sie hätten im Mittelalter durch ihre antideutsche Politik einen Erfolg der deutschen Kaiser auf den Kreuzzügen verhindert, was schließlich zum Verlust des Heiligen Landes geführt habe. Die späteren Historiker aus protestantischem Lager (Aventin, Johannes Carion, Sebastian Franck) führten das von Hutten vorgebrachte Argument fort, auch sie gaben den Päpsten die Schuld, daß den von deutschen Königen unternommenen Kreuzzügen kein dauerhafter Erfolg beschieden war und bezweifelten die theologische Berechtigung des Kreuzzugsablasses. Sie nahmen das Motiv Bernhards von Clairvaux „Schuld sind unsere Sünden“ wieder auf: Die Expansion der Türken sei eine Strafe Gottes, sie bestritten aber nicht den grundsätzlichen moralischen Anspruch aller Christen auf das Heilige Land.

\section{Ausblick}

Es galt zu zeigen, welche Wandlungen die Kreuzzugsidee zwischen dem Ende des 11. und dem des 15. Jahrhunderts durchgemacht hat. Anfangs eine bewaffnete Pilgerfahrt, auch in der Absicht unternommen, militärische Unterstützung für das an seiner Ostgrenze bedrängte oströmische Reich zu leisten, erwies sich der päpstlich verkündete Kreuzzugsablaß (vollkommener Ablaß für die Teilnahme) als Erfolgsmodell bei den Laien, so daß die Päpste der Versuchung nicht widerstanden, seit dem 13.Jahrhundert Kreuzzüge nicht nur gegen Heiden, sondern selbst gegen Ketzer und Christen auszurufen und den Teilnehmern die gleichen Ablässe zu versprechen. Damit desavouierten sie aber die Idee der Rückgewinnung des Heiligen Grabes und riefen eine nicht mehr verstummende Kreuzzugskritik auf den Plan, die theologisch und juristisch fundiert war. Nur hierokratisch orientierte Kanonisten lieferten für einen „Heiligen Glaubenskrieg“ eine allerdings gegenüber den werdenden alteuropäischen Nationalstaaten kaum noch durchzusetzende theologische Begründung. Die im Spätmittelalter etablierte römisch-rechtliche Norm von der Zulässigkeit der Gegengewalt (vim vi repellere fas est) wird zwar im kanonischen Straf- und Zivilprozeß mit der wichtigen Einschränkung „unter Wahrung der Verhältnismäßigkeit der Mittel“ (cum moderamine inculpate tutele), nicht aber im Völkerrecht zum Leitfaden. Für einen Angriffskrieg liefert die Norm von der Zulässigkeit der Gegengewalt keine Munition. Die franziskanische Idee der Mission ohne das Schwert blieb seit 1453 angesichts der anhaltenden Bedrohung durch die türkische Expansion auf dem Balkan vorerst eine idealistische Forderung. 


\section{Auswahlbibliographie}

Dieter Baner, Klaus Herbers, Nikolas Jaspert (Hrsg.), Jerusalem im Hoch- und Spätmittelalter (Campus Historische Studien 29, Frankfurt 2007).

Marcel Beck, Die geschichtliche Bedeutung der Kreuzzüge (Zürich 1953).

Wolfram Benziger, Zur Theorie von Krieg und Frieden in der italienischen Renaissance. Die Disputatio de pace et bello zwischen Bartolomeo Platina und Rodrigo Sánchez de Arévalo und andere anläßlich der Pax Paolina (Rom 1468) entstandene Schriften (Zürich 1996).

James A. Brundage, Medieval Canon Law and the Crusader (Madison, London 1969).

Giles Constable, The Second Crusade as seen by Contemporaries, in: Traditio 9 (1953) 162-188, wieder abgedruckt in: Giles Constable, Religious Life and Thought (London 1979) 125-146.

Kaspar Elm, Die Kreuzzüge. Kriege im Namen Gottes? (Köln 1996).

George B. Flabiff, Deus non vult. A Critic of the Third Crusade, in: Medieval Studies 9 (1947) 162-188.

Egon Flaig, „Heiliger Krieg“. Auf der Suche nach einer Typologie, in: HZ 285 (2007) 265-302.

Friedrich Heer, Die Kreuzzüge - gestern, heute, morgen (Frankfurt 1969).

Ernst-Dieter Hehl, Kirche und Krieg im 12. Jahrhundert. Studien zu kanonischem Recht und politischer Wirklichkeit (Monographien zur Geschichte des Mittelalters 19, Stuttgart 1980).

Dietrich Kurze, Krieg und Frieden im mittelalterlichen Denken, in: Heinz Duchbardt (Hrsg.), Zwischenstaatliche Friedenswahrung in Mittelalter und Früher Neuzeit (Köln, Wien 1991) 1-44.

Michael Menzel, Kreuzzugsideologie unter Innozenz III., in: Historisches Jahrbuch 120 (2000) 39-79.

Dieter Mertens, Europäischer Friede und Türkenkrieg im Spätmittelalter, in: Heinz Duchbardt (Hrsg.), Zwischenstaatliche Friedenswahrung in Mittelalter und Früher Neuzeit (Köln, Wien 1991) 45-90.

Erich Meuthen, Der Fall von Konstantinopel und der lateinische Westen, in: HZ 237 (1983) 1-35.

Joshua Prawer, The Latin Kingdom of Jerusalem: European Colonialism in the Middle Ages (London 1972).

Jonathan Riley-Smith, The Crusades (London 1987).

Jonathan Riley-Smith, Wozu Heilige Kriege? (Berlin 2003).

Frederick H. Russell, The Just War in the Middle Ages (Cambridge 1975).

Ludwig Schmugge, Die Kreuzzüge aus der Sicht humanistischer Geschichtsschreiber (Vorträge der Aeneas-Silvius-Stiftung an der Universität Basel XXI, Basel 1987).

Kenneth M. Setton (General editor), A History of the Crusades, 7 Bde. (Madison, London 1969-1989).

Elizabeth Sibbery, Criticism of Crusading, 1095-1274 (Oxford 1985). 
Palmer A. Throop, Criticism of the Crusade: a Study of Public Opinion and Crusade Propaganda (Amsterdam 1940).

John B. Williams, The Making of a Crusade: the Genoese anti-Moslim Attacks in Spain, 1146-1148, in: Journal of Medieval History 23 (1997) 29-53. 